\title{
Single port VATS mediastinal tumor resection: Taiwan experience
}

\author{
Ching-Yang Wu, Ming-Ju Heish, Ching-Feng Wu \\ Division of Thoracic and Cardiovascular Surgery, Department of Surgery, Chang Gung Memorial Hospital, Chang Gung University, Taoyuan, \\ Taiwan \\ Correspondence to: Ching-Feng Wu. Division of Thoracic and Cardiovascular Surgery, Department of Surgery, Chang Gung Memorial Hospital, \\ Chang Gung University, Taoyuan, Taiwan. Email: maple.bt88@gmail.com.
}

\begin{abstract}
Background: To present the technique of single-port video-assisted thoracoscopic mediastinal tumor resection, which includes limited thymectomy, extended thymectomy, cyst excision and posterior mediastinal tumor excision, and the early results of resection with the use of this technique.

Methods: Forty patients with mediastinal tumors were treated with single-port thoracoscopic mediastinal resection at Chang Gung Memorial Hospital between April 2014 and September 2015. The surgical intervention was performed through the fourth or fifth intercostal space at the anterior axillary line. A 5 or $10 \mathrm{~mm} 30$ degree video camera and working instruments were employed simultaneously at this incision site throughout the surgery.

Results: Among the 40 cases included in the final analysis, 10 extended thymectomies, 7 limited thymectomies, nine cyst excisions and 14 tumor excisions were performed successfully without the need for conversion. For the 40 patients who underwent single-port video-assisted thoracoscopic surgery (VATS), the mean operation time was $97.3 \pm 31.2 \mathrm{~min}$ and the average blood loss was $29.75 \pm 39.77 \mathrm{~mL}$. The average length of the incision wound was $3.22 \pm 0.79 \mathrm{~cm}$ and the average length of postoperative hospital stay was $3.72 \pm 1.63$ days. There were no mortalities and mobility was achieved within 30 days postoperatively.

Conclusions: Our preliminary report suggests that uniportal VATS for mediastinal tumor resection is a promising and safe technique within a short-term period.
\end{abstract}

Keywords: Minimally invasive surgery; single port video-assisted thoracoscopic surgery (VATS); extended thymectomy; mediastinum tumor

Submitted Nov 03, 2015. Accepted for publication Mar 02, 2016.

doi: $10.21037 /$ acs.2016.03.10

View this article at: http://dx.doi.org/10.21037/acs.2016.03.10

\section{Introduction}

Video-assisted thoracoscopic surgery (VATS) has been widely used for mediastinal tumor resections for over 20 years. Series of studies have demonstrated that its safety and oncological outcomes are not inferior to a median sternotomy (1-4). With the development of endoscopic instruments and VATS techniques, single-port VATS lung resection is being used for a growing number of applications (5-7). Limited clinical series, however, have described its use in mediastinal tumor resection $(8,9)$. Suda et al. first described their experience of infrasternal single port thymectomy (10). Although it may be useful for an anterior mediastinal tumor, it would be difficult to apply this technique to a middle or posterior mediastinal tumor. With reference to the single-port experience of Rivas et al. (7), we have gradually developed our transthoracic single-port procedure for mediastinal tumor resection, which will be described in detail.

\section{Methods and materials}

From April 2014 to September 2015, 40 consecutive patients underwent transthoracic single-port mediastinal tumor resection at the Department of General Thoracic Surgery at Chang Gung Memorial Hospital. The preoperative workup included chest radiography, chest computed tomography (CT) scan, spirometry and complete blood count. Age, 
gender, results of pulmonary function tests, operation time, blood loss, postoperative complications, length of hospital stay and tumor characteristics were collected from medical records. Surgical mortality was defined as death occurring during the same hospitalization or within 30 days after the operation. Indications for single-port thoracoscopic mediastinal tumor resection included (I) patients with a mediastinal tumor where no neoadjuvant therapy had been given; (II) patients with a mediastinal tumor that did not directly invade surrounding tissues, as seen on a CT scan; and (III) patients with a symptomatic mediastinal cyst or who had been referred for surgery for tissue sampling and tumor excision. For myasthenia gravis (MG) patients, we performed an extended thymectomy. Extended thymectomy was defined as the resection of the thymus gland as well as the right and left pericardial adipose tissue. Postoperative intensive care was required in MG patients who were refractory to medical control. All other patients were extubated in the operating room. All resected specimens were sent for pathologic staging and histologic typing was established according to the World Health Organization classification and Masaoka stage.

\section{Surgical technique}

The operative technique encountered in this procedure was as follows: under general anesthesia, the patients were intubated with a double lumen endotracheal tube. In the case of an anterior mediastinal tumor, the patient was placed in a 30 degree semi-supine position on the operating table with a roll placed beneath the shoulder and the ipsilateral arm was held abducted over a padded L-screen in order to expose the axilla (Figure 1A). If the patient had a posterior mediastinal tumor, a semi-prone position was used to place the patient on the table, with the contralateral hand placed beneath the neck and the ipsilateral side of the chest elevated by approximately 30 degrees. The ipsilateral arm was raised cranially to expose the axillary fossa (Figure $1 B$ ). If the tumor was located at the middle mediastinum, the lateral decubitus position was used. Using the positioning mentioned above, the target lesion could be directly viewed by the surgeons. The main tumor location determined the incision site. A $2-3 \mathrm{~cm}$ minithoracotomy was created initially in the fourth or fifth intercostal space at the anterior axillary line (Figure 1C). If the target lesion was located above the confluence of brachiocephalic vein and superior vena cava, or extended thymectomy was needed, a minithoracotomy on the fourth intercostal space was preferred over the fifth.

Otherwise, we often selected the fifth intercostal space for the incision site. A 30 degree 5 or $10 \mathrm{~mm}$ thoracoscope was then placed at the top of the wound. Rib resection or rib spreading was not needed in all procedures. The mediastinal specimen was retrieved using a plastic bag through the incision wound (Figure 1D). Sometimes, we encountered a tumor that was larger in size than the incision site. At that time, we gradually enlarged our incision site to allow for tumor retrieval. When we encountered mediastinal tumors that were tightly adherent to the lung parenchyma, a wedge resection was sometimes needed and the resection site was closed using an endostapler (Covidien ${ }^{\circledR}$, Mansfield, MA, USA or Ethicon Endo-Surgery ${ }^{\circledR}$, Inc., Cincinnati, OH, USA). An articulated endoscopic stapler was necessary for single-port surgery because it dramatically improved the accessibility of all target lesions. The dissection of the thymus proceeded along the innominate vein and division of the thymic veins with hemoclips (Covidien ${ }^{\circledR}$, Mansfield, MA, USA) or harmonic scalpel (Ethicon Endo-Surgery, Inc., Cincinnati, $\mathrm{OH}, \mathrm{USA})$. The bilateral thymus horns were dissected using an electric hook and lymph node grasper (Scanlan, USA). The operator and assistant stood together in the same site, facing the lesion. To obtain better visualization and traction angle, the surgical table could sometimes be rolled slightly forward or back. At the end of surgery, one chest tube or pig-tail was left for drainage. The drainage tube was placed at one end of the incision wound.

\section{Statistical analysis}

Continuous data are expressed as mean value with a range of standard deviation (SD). Data analysis was performed using SPSS software (version 18.0; SPSS, Chicago, IL, USA).

\section{Results}

Forty consecutive patients were included in the study group between April 2014 and September 2015. No patients converted to three-port VATS, thoracotomy or sternotomy. The study cohort consisted of 13 men and 27 women, with a mean age of $50.47 \pm 13.71$ years. Detailed clinical characteristics of all forty patients are listed in Table 1. Of the 40 patients, eight had refractory MG with thymoma or thymus hyperplasia, all of which received extended thymectomy. Nine patients had incidental findings of thymoma without a history of MG. Seven 

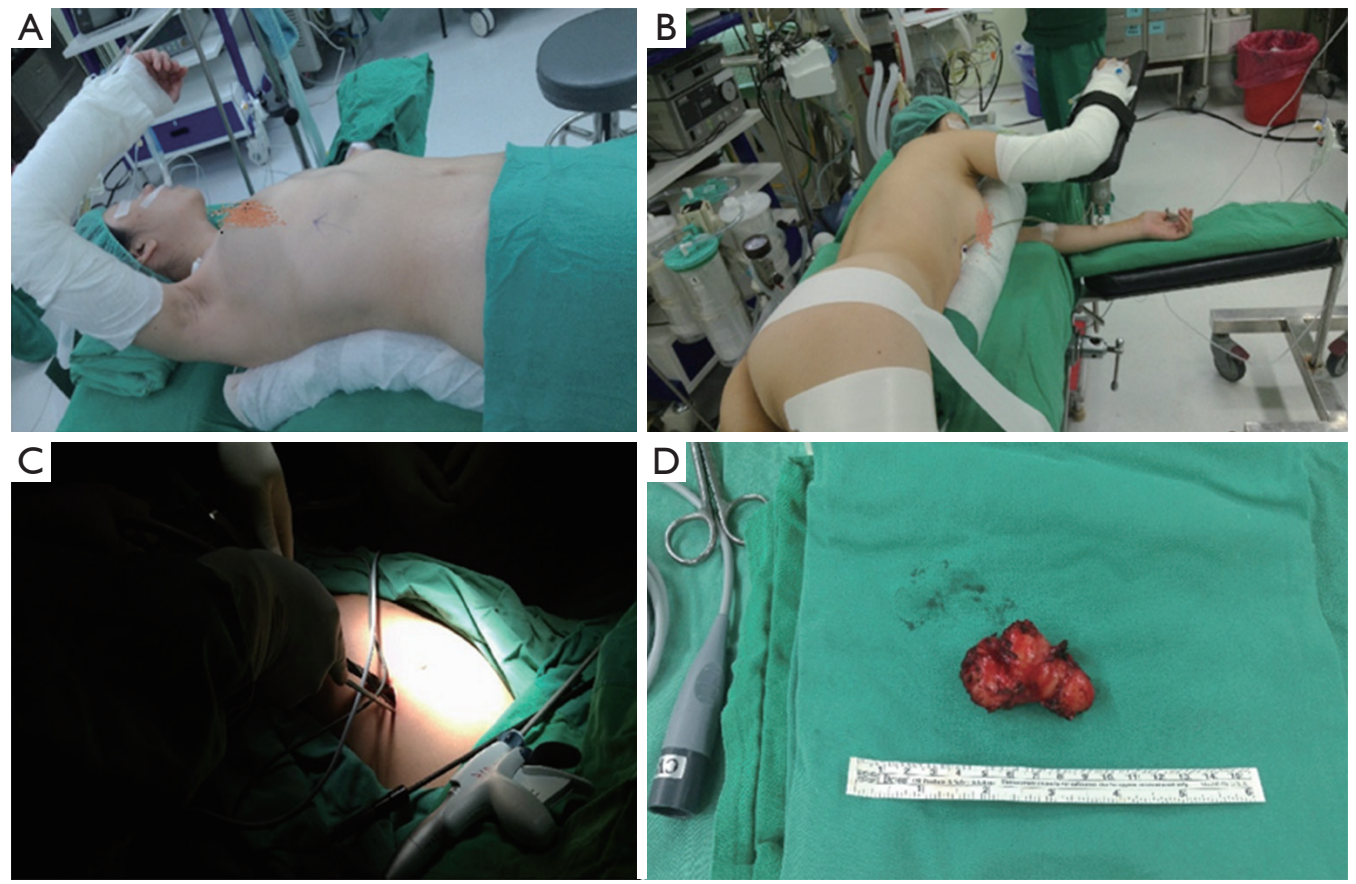

Figure 1 Operation position and instruments for mediastinal tumor resection. (A) Operation position for anterior mediastinal tumor; (B) operation position for posterior mediastinal tumor; (C) instruments for single-port video-assisted thoracoscopic mediastinal tumor resection; (D) posterior mediastinal tumor (Schwannoma).

\begin{tabular}{|c|c|}
\hline Characteristics & Patients \\
\hline \multicolumn{2}{|l|}{ Gender } \\
\hline Male & 27 \\
\hline Female & 13 \\
\hline Age (years) & $50.47 \pm 13.71$ \\
\hline BMI $\left(\mathrm{kg} / \mathrm{m}^{2}\right)$ & $25.43 \pm 4.02$ \\
\hline FEV1 (L) & $2.24 \pm 0.55$ \\
\hline \multicolumn{2}{|l|}{ Type of surgery } \\
\hline Extended thymectomy & 10 \\
\hline Limited thymectomy & 7 \\
\hline Cyst excision & 9 \\
\hline Tumor resection & 14 \\
\hline Wound length (cm) & $3.22 \pm 0.79$ \\
\hline Operative time (min) & $97.3 \pm 31.2$ \\
\hline Drainage duration (days) & $2.10 \pm 1.53$ \\
\hline Hospital stay (days) & $3.72 \pm 1.63$ \\
\hline
\end{tabular}

BMI, body mass index; FEV1, forced expiratory volume in one second. of these received limited thymectomy and two received extended thymectomy. Additionally, nine mediastinal cyst excisions and 15 tumor excisions were included in the study. The mean diameter of the resected tumor was $3.98 \pm 2.06 \mathrm{~cm}$ (range: 1.3 to $9 \mathrm{~cm}$ ), mean operation time was $97.3 \pm 31.2$ minutes, mean incision wound was $3.41 \pm 0.76 \mathrm{~cm}$ and the mean blood loss was $34.1 \pm 45.7 \mathrm{~mL}$. The mean postoperative drainage duration and hospital stay were $2.10 \pm 1.55$ and $3.74 \pm 1.64$ days, respectively. There was no occurrence of surgical morbidity or mortality in the study group. During the follow-up period (1-18 months), no recurrence of the tumor was noted.

\section{Discussion}

After Rocco et al. first published their single-port VATS experience since 2004 (5), the technique has been applied to different kinds of operations, including extended thymectomy, lobectomy, bilobectomy and sleeve lobectomy. However there have been few articles discussing single-port 
VATS mediastinum tumor resection to date. Advocates of single-port VATS have emphasized its benefits, including better cosmesis, less pain and faster recovery in the early postoperative period. These positive outcomes have also been observed in our limited experience $(1,11,12)$.

The incision design is extremely important to ensure that the entire mediastinum space is accessible. The best choice for the incision site is at the fourth or fifth intercostal space. The fourth intercostal space is especially suitable for extended thymectomy or when the tumor's location is above the confluence of the superior vena cava and brachiocephalic vein. In addition, a bigger tumor $(>5 \mathrm{~cm})$ might hinder the surgeon's field of vision. In this situation, opening the mediastinal pleural cavity first might be necessary allowing the surgeons to have more space to manipulate the mediastinal tumor. We often use a harmonic scalpel and endostapler during the operation. The use of an energy device and endostapler could help shorten operative times and prevent small vessel bleeding when the tumor is tightly adherent to the lung parenchyma.

It is also important to discuss the impact of pain on patients following single-port surgery. A small wound incision and a smaller chance of affecting the intercostal nerves are likely to cause a lesser degree of postoperative pain to patients. However, pain is subjective and may be affected by a number of factors, including operative time, drainage tube size and post-operative analgesic regimens. In fact, we considered avoiding the use chest tubes in a simple mediastinal tumor resection, such as for a mediastinal cyst, as this might help to reduce postoperative pain. However, for very large mediastinal tumors or tumors that adhere to surrounding organs, the use of chest tubes is often been necessary. Most of the patients who received single-port VATS could ambulate on the ward on day 1 postoperatively. In the future, we aim to enroll more patients to validate our clinical findings.

In our limited experience, all the dissections were performed with the surgeon's dominant hand at the bottom of the working port, with the non-dominant hand keeping the operative field clean. As long as the instruments are used from the same working port, operative time will gradually shorten without surgical complications, as we become more experienced with this approach. It is important to note that there were no patients converted from singleport surgery to multiple-port VATS or open thoracotomy, which further suggests that single-port surgery is a safe and feasible procedure for the removal of non-invasive mediastinal tumors. Safety is the first consideration when a new procedure is applied to patients.

\section{Conclusions}

Our preliminary report revealed that single-port VATS for non-invasive mediastinal tumor resection is a safe and reliable method. Studies on the long-term oncologic outcomes associated with this approach are required to further confirm its benefits.

\section{Acknowledgements}

None.

\section{Footnote}

Conflicts of Interest: The authors have no conflicts of interest to declare.

\section{References}

1. Rückert JC, Walter M, Müller JM. Pulmonary function after thoracoscopic thymectomy versus median sternotomy for myasthenia gravis. Ann Thorac Surg 2000;70:1656-61.

2. Shigemura $N$, Shiono $H$, Inoue $M$, et al. Inclusion of the transcervical approach in video-assisted thoracoscopic extended thymectomy (VATET) for myasthenia gravis: a prospective trial. Surg Endosc 2006;20:1614-8.

3. Yim AP. Video-assisted thoracoscopic resection of anterior mediastinal masses. Int Surg 1996;81:350-3.

4. Yim AP, Kay RL, Ho JK. Video-assisted thoracoscopic thymectomy for myasthenia gravis. Chest 1995;108:1440-3.

5. Rocco G, Martin-Ucar A, Passera E. Uniportal VATS wedge pulmonary resections. Ann Thorac Surg 2004;77:726-8.

6. Berlanga LA, Gigirey O. Uniportal video-assisted thoracic surgery for primary spontaneous pneumothorax using a single-incision laparoscopic surgery port: a feasible and safe procedure. Surg Endosc 2011;25:2044-7.

7. Gonzalez-Rivas D, Paradela M, Fieira E, et al. Singleincision video-assisted thoracoscopic lobectomy: initial results. J Thorac Cardiovasc Surg 2012;143:745-7.

8. Wu CF, Gonzalez-Rivas D, Wen CT, et al. Single-port video-assisted thoracoscopic mediastinal tumour resection. Interact Cardiovasc Thorac Surg 2015;21:644-9.

9. Wu CF, Gonzalez-Rivas D, Wen CT, et al. Comparative 
Short-Term Clinical Outcomes of Mediastinum Tumor Excision Performed by Conventional VATS and SinglePort VATS: Is It Worthwhile? Medicine (Baltimore) 2015;94:e1975.

10. Suda T, Ashikari S, Tochii S, et al. Single-incision subxiphoid approach for bilateral metastasectomy. Ann Thorac Surg 2014;97:718-9.

11. Shiono H, Kadota Y, Hayashi A, et al. Comparison of outcomes after extended thymectomy for myasthenia gravis: bilateral thoracoscopic approach versus

Cite this article as: Wu CY, Heish MJ, Wu CF. Single port VATS mediastinal tumor resection: Taiwan experience. Ann Cardiothorac Surg 2016;5(2):107-111. doi: 10.21037/ acs.2016.03.10 sternotomy. Surg Laparosc Endosc Percutan Tech 2009;19:424-7.

12. Zahid I, Sharif S, Routledge T, et al. Video-assisted thoracoscopic surgery or transsternal thymectomy in the treatment of myasthenia gravis? Interact Cardiovasc Thorac Surg 2011;12:40-6. 